\title{
Mapping the non-structural transmembrane proteins of SARS-CoV-2
}

\author{
Sunil Thomas
}

Lankenau Institute for Medical Research, Wynnewood, PA-19096, USA.

E-mail: suntom2@gmail.com

\begin{abstract}
Severe acute respiratory syndrome coronavirus 2 (SARS-CoV-2) responsible for the disease COVID-19 has wreaked havoc on the health and economy of humanity. In addition, the disease is observed in domestic and wild animals. The disease has impacted directly and indirectly every corner of the planet. Currently, there are no vaccines and effective therapies for COVID-19. SARS-CoV-2 is an enveloped virus with a singlestranded RNA genome of $29.8 \mathrm{~kb}$. More than two-thirds of the genome comprises Orf1ab encoding 16 non-structural proteins (nsps) followed by mRNAs encoding structural proteins, spike (S), envelop (E), membrane $(M)$, and nucleocapsid $(N)$. These genes are interspaced with several accessory genes (open reading frames [Orf] 3a, 3b, 6, 7a, 7b, $8,9 b, 9 c$ and 10). The functions of these proteins are of particular interest for understanding the pathogenesis of SARS-CoV-2. Several of the nsps (nsp3, nsp4, nsp6) and Orf3a are transmembrane proteins involved in regulating the host immunity, modifying host cell organelles for viral replication and escape and hence considered drug targets. In this paper we report mapping the transmembrane structure of the nonstructural proteins of SARS-CoV-2.
\end{abstract}

Keywords: SARS-CoV-2, COVID-19, coronavirus, non-structural proteins, nsps, nsp3, nsp4, nsp6, Orf1ab, Orf3a, endoplasmic reticulum, double membrane vesicle. 


\section{INTRODUCTION}

The coronavirus-19 disease (COVID-19) caused by severe acute respiratory syndrome coronavirus 2 (SARS-CoV-2) in late 2019 is responsible for the pandemic and is a major public health concern. The disease has resulted in mortality, massive hospitalizations, lockouts, economic loss, employment layoffs, school and college closings, and have impacted all the countries. The SARS-CoV-2 virus not only infects humans, but also companion and wild animals. Even after a year, there are no effective medicines or vaccines to protect against the disease (Thomas, 2020). The only effective strategies to decrease the incidence of the disease is social distancing, use of masks and lockdowns in seriously affected areas.

People with diabetes are at risk of the disease. As yet we do not know why the virus is highly successful in causing the pandemic within 3 months of its first report (Thomas, 2020). Understanding the structure and function of the proteins of SARS-CoV-2 will lead to the development of effective vaccines and drugs to protect against the virus.

The structural proteins of SARS-CoV-2 include, membrane glycoprotein (M), envelope protein $(E)$, nucleocapsid protein $(N)$ and the spike protein $(S)$. SARS-CoV-2 contains a 29.8-kb single-stranded RNA genome wrapped in a helical nucleocapsid composed of multiple copies of $\mathrm{N}$ protein, which in turn is surrounded by an envelope containing $S$ glycoprotein, M glycoprotein, and a small E protein. The viral gene order is similar to that in other known coronaviruses, with the first two open reading frames ( $1 a$ and $1 b$ ) encoding the viral replicase and the downstream mRNAs encoding the structural proteins. Using bioinformatics, we previously showed that the M proteins of SARS-CoV-2 resembles the sugar transporters that may be involved in functions favorable to the virus (Thomas, 2020). The non-structural proteins (nsps) of SARS-CoV-2 are involved in inhibiting innate immunity and also induce virus replication. Two overlapping Orfs, Orf1a and Orf1b, are translated from the positive-strand genomic RNA and generate continuous polypeptides, which are cleaved into a total of $16 \mathrm{nsps}$. The genes coding for the structural proteins are interspaced with several accessory genes including the open reading frames (Orf) 3a, 
$3 b, 6,7 a, 7 b, 8,9 b, 9 c, 10)$. The functions of these proteins are of particular interest for understanding the pathogenesis of SARS-CoV-2 (Yoshimoto, 2020; Gordon et al. 2020).

To evade detection by host innate immune sensors, viruses that replicate in the cytoplasm compartmentalize their genome transcription in organelle-like structures, thereby protecting the virus against host cell defenses and increasing the replication efficiency (Belov and van Kuppeveld, 2012). The transmembrane nsp3, nsp4 and nsp6 are known to rearrange endoplasmic reticulum (ER) membranes thereby inducing curvature of the ER membrane, essential for virus replication. Lack of antiviral therapies is the paucity of knowledge regarding the $\beta$-coronavirus-host cell interface (Ghosh et al. 2020). In this paper we provide information on the transmembrane and lumen domains of the nsps of SARS-CoV-2.

\section{MATERIALS AND METHODS}

\section{SARS-CoV-2 transmembrane non-structural protein structure}

The transmembrane non-structural protein sequences of the SARS-CoV-2 were downloaded from the NCBI (https://www.ncbi.nlm.nih.gov/protein/) protein database. The transmembrane non-structural proteins of SARS-COV-2 include nsp3 (Accession No. YP_009725299), nsp4 (Accession No. YP_009725300), nsp6 (Accession No. YP_009725302) and Orf3a (Accession No. BCl50534).

\section{Protein modeling}

For a comprehensive understanding of biological systems of how proteins complexes and networks operate a detailed description of the interactions of proteins and the overall quaternary structure is essential. Residue-based diagrams of proteins, also called snake diagrams or protein plots, are 2-D representations of a protein sequence that contain information about properties such as secondary structure (Skrabanek et al. 2003). To determine a snake diagram model of a protein we used Protter (http://wlab.ethz.ch/protter). Protter is an interactive and customizable web-based application that enables the integration and visualization of both annotated and predicted 
protein sequence features together with experimental proteomic evidence for peptides and posttranslational modifications onto the transmembrane topology of a protein. It allows users to choose from numerous annotation sources, integrate their own proteomics data files, select the best-suited peptides for targeted quantitative proteomics applications, and export publication-quality illustrations (Omasits et al. 2014).

For the three dimensional homology modeling we employed the iterative threading assembly refinement (I-TASSER) (https://zhanglab.ccmb.med.umich.edu/l-TASSER/) with default settings. The protein sequences of SARS-CoV-2 was entered in FASTA format.

\section{Multiple sequence alignments}

Multiple sequence alignments (MSAs) are essential in bioinformatics analyses that involve comparing homologous sequences (Thompson et al. 1994). ClustalW2 is a server for MSA that is also used for phylogenetic tree analysis. Multiple sequence alignments between the ORF3a protein of SARS-CoV-2 and that of SARS-CoV was performed using the ClustalW2 server (http:/www.ebi.ac.uk/tools/msa/clustalW2/).

\section{RESULTS}

As yet there are no effective therapies and vaccines for COVID-19. To meet the increasing demand for the treatment of COVID-19 there is a need to accelerate novel antiviral drug development as quickly as possible. Target-based drug development may be a promising approach to achieve this goal (Liu et al. 2021). Identifying molecular targets could lead to development of medications that protect against SARS-CoV-2 virus.

Coronaviruses, including SARS-CoV-2 that replicate in the cytoplasm compartmentalize their genome transcription in organelle-like structures thereby protecting the virus against host cell defenses and increasing the replication efficiency (Santerre et al. 2020). We previously mapped the structural proteins of SARS-CoV-2 and demonstrated that the M protein the virus resembles the sugar transporter SemiSWEET (Thomas, 2020). 
The nsps are critical elements of the replication and transcription complex (RTC), as well as immune system evasion. Through hijacking the endoplasmic reticulum (ER) membrane, nsps help the virus establish the RTC (Santerre et al. 2020). The structure and function of nsps of SARS-CoV-2 is similar to SARS-CoV. In SARS-CoV, the primary structures of the three nsps- nsp3, nsp4, and nsp6, contain hydrophobic stretches, and these proteins are predicted to be integral membrane proteins. Hence, they are likely to function in anchoring the replication complexes to the lipid bilayer (Oostra et al. 2007).

Using bioinformatics, we mapped the transmembrane nsps of SARS-CoV-2. We initially mapped the Orf1ab. The Orf1ab codes for $16 \mathrm{nsps}$. The Orf1ab is cleaved into $16 \mathrm{nsps}$. Analysis of the Orf1ab by Protter showed the three transmembrane nsps- nsp3, nsp4, nsp6 (Fig. 1).

We modeled each of the transmembrane nsps. Luminal loops of the transmembrane nsps are essential for rearranging ER membranes thereby inducing curvature of the ER membrane. The nsp3 has two transmembrane domains and a luminal domain in the ER lumen. The nsp3 snake diagram, 3D model and the various domains (cytoplasmic, transmembrane, luminal) are shown in Fig. 2.

The nsp4 is the largest transmembrane nsp and the second largest transmembrane protein of SARS-CoV-2 after the Spike protein. The nsp4 protein has four transmembrane domains and a large luminal domain in the ER lumen between the first two transmembrane domains. Nsp4 also has a smaller luminal domain in the ER lumen between the third and four transmembrane domains (Fig. 3).

The nsp6 has six transmembrane domains and two small luminal domains between the third and fourth as well as fifth and sixth transmembrane in the ER lumen (Fig. 4).

The ORF3a of SARS-CoV-2 is also a transmembrane protein. However, it was difficult to model it by Protter. The structure of Orf3a of SARS CoV-2 is similar to SARS-CoV and has around $73 \%$ homology as determined by ClustalW (Fig. 5). Hence, we used Orf3a of 
SARS-CoV as a template to model the snake diagram. The Orf3a has three transmembrane domains and a long and short luminal domain jutting into the ER lumen (Fig. 5).

The transmembrane domains of the nsps of SARS-CoV-2 is responsible for inhibiting the host immunity as well as increasing the replication efficiency of the virus. In this paper we have mapped the transmembrane nsps of SARS-CoV-2 and they could be used as a target to inhibit virus replication.

\section{DISCUSSION}

The COVID-19 pandemic caused by the SARS-CoV-2 virus has immobilized the world. It is the most severe pandemic of the twentieth century. As of the first week of December 2020, the virus has infected 63 million people, with 1.5 million deaths world-wide. The disease is more severe in old people compared to children and young adults. Currently there are no therapies and vaccines for the disease; hence, there is an urgent need to develop effective therapies and vaccines for the deadly disease.

The four structural proteins of SARS-CoV-2 are spike (S), envelope (E), membrane (M), and nucleocapsid $(\mathrm{N})$ proteins. In addition, the virus consists of a large polyprotein, Orf1ab that proteolytically cleave to form 16 non-structural proteins (nsps). There are also accessory proteins: Orf3a, Orf3b, Orf6, Orf7a, Orf7b, Orf8, Orf9b, Orf9c and Orf10. Although accessory proteins have been viewed as dispensable for viral replication in vitro, some have been shown to play an important role in virus-host interactions in vivo (Yoshimoto, 2020; Gordon et al. 2020).

The entry of the SARS-CoV-2 into cells starts when the spike glycoprotein expressed on the viral envelope binds to ACE2 receptor of the host cell. The virus enters the cells through endocytosis process, which is possibly facilitated, via a $\mathrm{pH}$ dependent endosomal cysteine protease cathepsins. Once inside the cells, SARS-CoV-2 exploits the endogenous transcriptional machinery of host cells to replicate and spread inside the cell. 
The virus activates or hijack the intracellular pathways of the host in favor of its replication (Sureda et al. 2020).

Knowledge on the structure of the structural proteins of SARS-CoV-2 is essential to the development of vaccines. Most of the vaccines that protect against SARS-CoV-2 developed in the laboratory are based on the S protein (Gu et al. 2020; vanDoremalan et al. 2020; McKay et al. 2020). Several commercial entities are also developing the vaccines based on the Spike mRNA, adenovector or recombinant protein.

In a previous paper we showed the structures of the structural proteins of SARS-CoV-2. In silico analysis showed that the M protein of SARS-CoV-2 resembled the prokaryotic sugar transporter SemiSWEET (Thomas, 2020). The nsps are involved in inhibiting host innate immunity, inducing RNA replication and virus exit. They are potential drug targets. However, the transmembrane domains of these nsps are not clearly documented. In this paper we report the domains of the nsps that may be targets for potential drug candidates.

The first nsps encoded by Orf1a/Orf1ab are papain-like proteinase (PL proteinase, nsp3) and 3-chymotrypsin-like proteinase (3CLPro protease). The PL proteinase nsp3 cleaves nsps 1 to 3 and the 3CLPro proteinase slices the C-terminus from nsp4 to nsp16. Nsp3 is the largest element of the RTC. In addition to cleaving, nsp3 alters cytokine expression to decrease the host innate immune response. Nsp3, nsp4, and nsp6 form a complex to induce double-membrane vesicles (DMV) (Rohaim et al. 2020).

Replication and transcription of the virus happen within a replication/transcription complex (RTC) encoded by the virus with nsps as primary constituents. Nearly all RTC elements are encoded by the large replicase gene that consists of Orf1a and Orf1ab. The early formation of the RTC is an essential step in the SARS-CoV-2 life cycle to safeguard viral genome replication and to synthesize subgenomic mRNA (Santerre et al. 2020).

SARS-CoV-2 are positive-stranded RNA viruses that replicate their unusually large genomes in the host cell's cytoplasm. RNA replication is supported by an elaborate virus- 
induced network of transformed endoplasmic reticulum (ER) membranes. Doublemembrane vesicles (DMVs) are the central hubs for viral RNA synthesis. The DMV's interior accumulates double-stranded (ds) RNA, presumably intermediates of viral genome replication and subgenomic mRNA synthesis. DMVs may offer a favorable microenvironment for viral RNA synthesis and may shield viral RNA from innate immune sensors that are activated by dsRNA. The coronavirus genome needs to be packaged by the cytosolic nucleocapsid $(\mathrm{N})$ protein before being targeted to virus assembly sites on secretory pathway membranes (Wolff et al. 2020).

Until recently it was not understood how the newly synthesized genomes and messenger RNAs can travel from the sealed replication compartments to the cytosol to ensure their translation and the assembly of progeny virions. Wolff et al. (2020) showed that a molecular pore spans the DMVs and it allows the export of newly synthesized viral RNA from the DMVs to the cytosol. They also demonstrated that the nsp3, nsp4 and nsp6 are components of the pore. Several nsp3 domains, including the conserved $\mathrm{N}$-terminal ubiquitin-like domain 1 (Ubl1) binds single-stranded RNA and the $\mathrm{N}$ protein that also interact with viral RNA. The double-membrane-spanning molecular pore may constitute the exit pathway for coronaviral RNA products from the DMV's interior toward the cytosol, with the large and multifunctional nsp3 being its central component. In this paper we map the ER luminal domain of nsp3, nsp4 and nsp6 that may be involved in DMV formation.

Interferons (IFNs) are cytokines with strong antiviral activities and is the first line of defense against invading pathogens. Multiple nsps are involved in inhibiting IFN-I production. The nsp6 binds TANK binding kinase 1 (TBK1) to suppress interferon regulatory factor 3 (IRF3) phosphorylation, nsp13 binds and blocks TBK1 phosphorylation, and Orf6 binds importin Karyopherin a 2 (KPNA2) to inhibit IRF3 nuclear translocation. SARS-CoV-2 nsp1 and nsp6 suppress IFN-I signaling more efficiently than SARS-CoV and Middle East respiratory syndrome coronavirus (MERS-CoV) (Xia et al. 2020).

The Orf3a protein is expressed abundantly in infected and transfected cells, which localizes to intracellular and plasma membranes. (Hassan et al. 2020). ORF3a induce 
apoptosis of cells mediated through caspase 3 (Ren et al. 2020). Issa et al. (2020) identified six functional domains (I to VI) in the SARS-CoV-2 Orf3a protein. The functional domains were linked to virulence, infectivity, ion channel formation, and virus release. Orf 3a may also be involved in vesicle trafficking (Gordon et al. 2020). The sequence of Orf3a of SARS-CoV-2 is $73 \%$ similar to that of SARS. As it was difficult to model the Orf3a sequence of SARS-CoV-2 by Protter, we used the corresponding sequence of SARS. Based on our analysis, the Orf3a has three transmembrane domains and a long and short luminal domain jutting into the ER lumen.

Overall, this paper maps the structure of the nsps that modify the ER to DMVs so as induce replication and further exit the host cell. How the SARS-CoV-2 regulate the host cell to hide in DMVs and replicate will lead to the development of therapies that treat COVID-19 (Santerre et al. 2020). 


\section{REFERENCES}

Belov GA, van Kuppeveld FJ. (+)RNA viruses rewire cellular pathways to build replication organelles. Curr Opin Virol. 2012 Dec;2(6):740-7. doi: 10.1016/j.coviro.2012.09.006.

Ghosh S, Dellibovi-Ragheb TA, Kerviel A, Pak E, Qiu Q, Fisher M, Takvorian PM, Bleck C, Hsu VW, Fehr AR, Perlman S, Achar SR, Straus MR, Whittaker GR, de Haan CAM, Kehrl J, Altan-Bonnet G, Altan-Bonnet N. $\beta$-Coronaviruses use lysosomes for egress instead of the biosynthetic secretory pathway. Cell. 2020 Oct 27:S0092-8674(20)31446X. doi: 10.1016/j.cell.2020.10.039.

Gordon DE, Jang GM, Bouhaddou M, et al. A SARS-CoV-2 protein interaction map reveals targets for drug repurposing. Nature. 2020 Jul;583(7816):459-468. doi: 10.1038/s41586-020-2286-9.

Gu H, Chen Q, Yang G, He L, Fan H, Deng YQ, Wang Y, Teng Y, Zhao Z, Cui Y, Li Y, Li XF, Li J, Zhang NN, Yang X, Chen S, Guo Y, Zhao G, Wang X, Luo DY, Wang H, Yang X, Li Y, Han G, He Y, Zhou X, Geng S, Sheng X, Jiang S, Sun S, Qin CF, Zhou Y. Adaptation of SARS-CoV-2 in BALB/c mice for testing vaccine efficacy. Science. 2020 Sep 25;369(6511):1603-1607. doi: 10.1126/science.abc4730.

Hassan SS, Choudhury PP, Basu P, Jana SS. Molecular conservation and differential mutation on ORF3a gene in Indian SARS-CoV2 genomes. Genomics. 2020 Sep;112(5):3226-3237. doi: 10.1016/j.ygeno.2020.06.016.

Issa E, Merhi G, Panossian B, Salloum T, Tokajian S. SARS-CoV-2 and ORF3a: Nonsynonymous Mutations, Functional Domains, and Viral Pathogenesis. mSystems. 2020 May 5;5(3):e00266-20. doi: 10.1128/mSystems.00266-20.

Liu XH, Zhang X, Lu ZH, Zhu YS, Wang T. Potential molecular targets of nonstructural proteins for the development of antiviral drugs against SARS-CoV-2 infection. Biomed Pharmacother. 2020 Nov 17;133:111035. doi: 10.1016/j.biopha.2020.111035.

McKay PF, Hu K, Blakney AK, Samnuan K, Brown JC, Penn R, Zhou J, Bouton CR, Rogers P, Polra K, Lin PJC, Barbosa C, Tam YK, Barclay WS, Shattock RJ. Selfamplifying RNA SARS-CoV-2 lipid nanoparticle vaccine candidate induces high neutralizing antibody titers in mice. Nat Commun. 2020 Jul 9;11(1):3523. doi: 10.1038/s41467-020-17409-9.

Omasits U, Ahrens $\mathrm{CH}$, Müller S, Wollscheid B. Protter: interactive protein feature visualization and integration with experimental proteomic data. Bioinformatics 2014; 30:884-6. doi: 10.1093/bioinformatics/btt607.

Ren Y, Shu T, Wu D, Mu J, Wang C, Huang M, Han Y, Zhang XY, Zhou W, Qiu Y, Zhou $X$. The ORF3a protein of SARS-CoV-2 induces apoptosis in cells. Cell Mol Immunol. 2020 Aug;17(8):881-883. doi: 10.1038/s41423-020-0485-9. 
Rohaim MA, El Naggar RF, Clayton E, Munir M. Structural and functional insights into non-structural proteins of coronaviruses. Microb Pathog. 2020 Nov 23:104641. doi: 10.1016/j.micpath.2020.104641.

Santerre M, Arjona SP, Allen CN, Shcherbik N, Sawaya BE. Why do SARS-CoV-2 NSPs rush to the ER? J Neurol. 2020 Sep 1:1-10. doi: 10.1007/s00415-020-10197-8.

Skrabanek L, Campagne F, Weinstein $\mathrm{H}$. Building protein diagrams on the web with the residue-based diagram editor RbDe. Nucleic Acids Res 2003; 31: 3856-3858. doi: 10.1093/nar/gkg552.

Sureda A, Alizadeh J, Nabavi SF, Berindan-Neagoe I, Cismaru CA, Jeandet P, Łos MJ, Clementi E, Nabavi SM, Ghavami S. Endoplasmic reticulum as a potential therapeutic target for covid-19 infection management? Eur J Pharmacol. 2020 Sep 5;882:173288. doi: 10.1016/j.ejphar.2020.173288.

Thomas S. The structure of the membrane protein of SARS-CoV-2 resembles the sugar transporter SemiSWEET. Pathog Immun. 2020 Oct 19;5(1):342-363. doi: 10.20411/pai.v5i1.377.

Thompson JD, Higgins DG, Gibson TJ. CLUSTAL W: improving the sensitivity of progressive multiple sequence alignment through sequence weighting, position-specific gap penalties and weight matrix choice. Nucleic Acids Res 1994; 22:4673-80. doi: 10.1093/nar/22.22.4673.

van Doremalen N, Lambe T, Spencer A, Belij-Rammerstorfer S, Purushotham JN, et al. ChAdOx1 nCoV-19 vaccine prevents SARS-CoV-2 pneumonia in rhesus macaques. Nature. 2020 Oct;586(7830):578-582. doi: 10.1038/s41586-020-2608-y.

Wolff G, Limpens RWAL, Zevenhoven-Dobbe JC, Laugks U, Zheng S, de Jong AWM, Koning RI, Agard DA, Grünewald K, Koster AJ, Snijder EJ, Bárcena M. A molecular pore spans the double membrane of the coronavirus replication organelle. Science. 2020 Sep 11;369(6509):1395-1398. doi: 10.1126/science.abd3629.

Xia H, Cao Z, Xie X, Zhang X, Chen JY, Wang H, Menachery VD, Rajsbaum R, Shi PY. Evasion of Type I Interferon by SARS-CoV-2. Cell Rep. 2020 Oct 6;33(1):108234. doi: 10.1016/j.celrep.2020.108234. Epub 2020 Sep 19.

Yoshimoto FK. The proteins of Severe Acute Respiratory Syndrome Coronavirus-2 (SARS CoV-2 or n-COV19), the cause of COVID-19. Protein J. 2020 Jun;39(3):198-216. doi: 10.1007/s10930-020-09901-4. 


\section{ACKNOWEDGEMENTS}

The author acknowledges Abraham Thomas Foundation for providing the resources for this work.

\section{CONFLICT OF INTEREST}

The author declares no conflict of interest. 


\section{FIGURES}

Fig.1. The topology of Orf1ab (snake diagram) determined using Protter. The Orf1ab is cleaved into 16 nsps. Analysis of the Orf1ab shows the three transmembrane nonstructural proteins-nsp3, nsp4, nsp6. The 12 transmembrane domains of the nsps are shown in figures $2-4$.

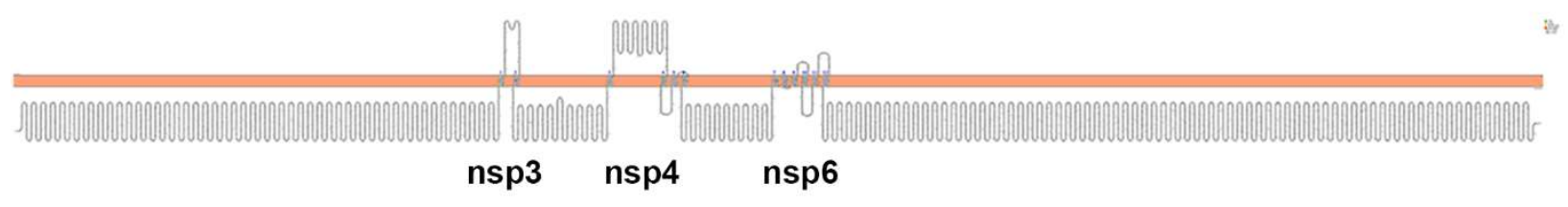

Fig.2. The topology of nsp3 of SARS-CoV-2. (A) Membrane topology of nsp3 determined using Protter. (B) The domains (cytoplasmic, transmembrane, luminal) of nsp3. (C) The predicted nsp3 protein structure of SARS-CoV-2 (ribbon diagram) determined using the software I-TASSER. The amino acid sequence from 1201-1945 of nsp3 is used for modeling the protein.

A

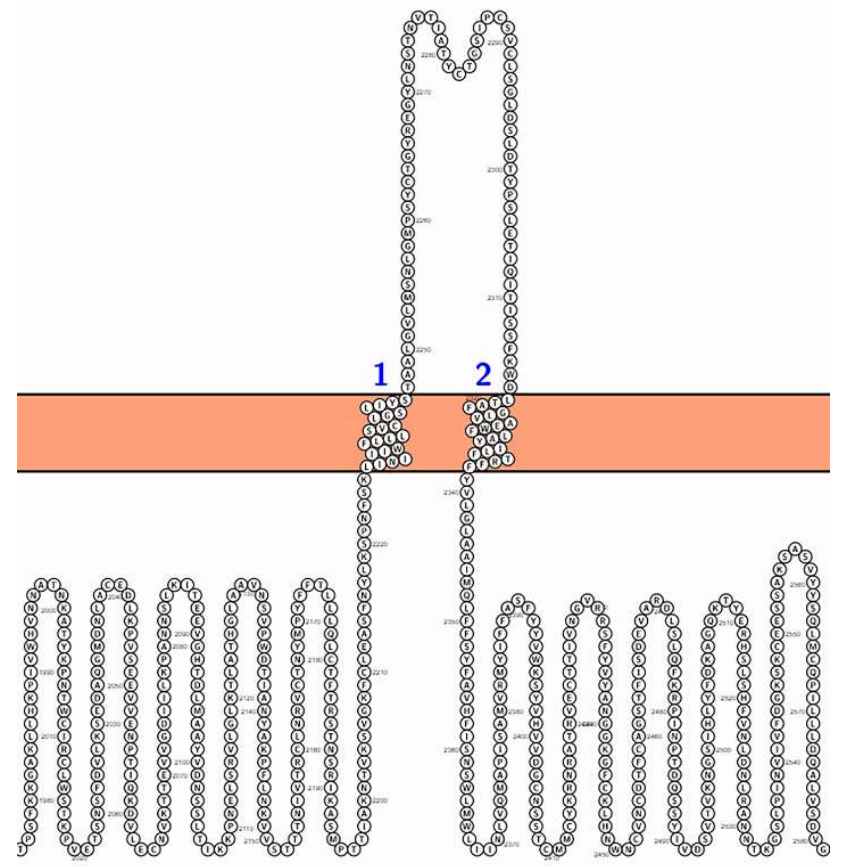


B

1 aptkvtfgdd tvievqgyks vnitfelder idkvlnekcs aytvelgtev nefacvvada

61 viktlqpvse lltplgidld ewsmatyylf desgefklas hmycsfyppd edeeegdcee

121 eefepstqye ygteddyqgk plefgatsaa lqpeeeqeed wldddsqqtv gqqdgsednq

181 tttiqtivev qpqlemeltp vvqtievnsf sgylkltdnv yiknadivee akkvkptvvv

241 naanvylkhg ggvagalnka tnnamqvesd dyiatngplk vggscvlsgh nlakhclhvv

301 gpnvnkgedi qllksayenf nqhevllapl lsagifgadp ihslrvevdt vrtnvylavf

361 dknlydklvs sflemksekq veqkiaeipk eevkpfites kpsveqrkqd dkkikacvee

421 vtttleetkf ltenlllyid ingnlhpdsa tlvsdiditf lkkdapyivg dvvqegvlta

481 vviptkkagg ttemlakalr kvptdnyitt ypgqglngyt veeaktvlkk cksafyilps

541 iisnekqeil gtvswnlrem lahaeetrkl mpvcvetkai vstiqrkykg ikiqegvvdy

601 garfyfytsk ttvaslintl ndlnetlvtm plgyvthgln leeaarymrs lkvpatvsvs

661 spdavtayng yltsssktpe ehfietisla gsykdwsysg qstqlgiefl krgdksvyyt

721 snpttfhldg evitfdnlkt llslrevrti kvfttvdnin lhtqvvdmsm tygqqfgpty

781 ldgadvtkik phnshegktf yvlpnddtlr veafeyyhtt dpsflgryms alnhtkkwky

841 pqvngltsik wadnncylat alltlqqiel kfnppalqda yyrarageaa nfcalilayc

901 nktvgelgdv retmsylfqh anldsckrvl nvvcktcgqq qttlkgveav mymgtlsyeq

$961 \mathrm{fkkgvqipct}$ cgkqatkylv qqespfvmms appaqyelkh gtftcaseyt gnyqcghykh

1021 itsketlyci dgalltksse ykgpitdvfy kensytttik pvtykldgvv cteidpkldn

1081 yykkdnsyft eqpidlvpnq pypnasfdnf kfvednikfa ddlnqltgyk kpasrelkvt

1141 ffpdlngdvv aidykhytps fkkgakllhk pivwhvnnat nkatykpntw circlwstkp

1201 vetsnsfdvl ksedaqgmdn lacedlkpvs eevvenptiq kdvlecnvkt tevvgdiilk

1261 pannslkite evghtdlmaa yvdnssltik kpnelsrvlg lktlathgla avnsvpwdti

1321 anyakpflnk vvstttnivt rclnrvctny mpyfftlllq letftrstns rikasmptti

1381 akntvksvgk fcleasfnyl kspnfsk lin iiiwflllsv clgsliys ta algvlmsnlg

1441 mpsyctgyre gylnstnvti atyctgsipc svclsgldsl dtypsletiq itissfkwd 1

1501 tafglvaewf layilftrff yvlglaaimq lffsyfavhf isnswlmwli inlvqmapis

1561 amvrmyiffa sfyyvwksyv hvvdgcnsst cmmcykrnra trvecttivn gvrrsfyvya

1621 nggkgfcklh nwncvncdtf cagstfisde vardlslqfk rpinptdqss yivdsvtvkn

1681 gsihlyfdka gqktyerhsl shfvnldnlr anntkgslpi nvivfdgksk ceessaksas

1741 vyysqlmcqp illldqalvs dvgdsaevav kmfdayvntf sstfnvpmek lktlvataea

1801 elaknvsldn vlstfisaar qgfvdsdvet kdvveclkls hqsdievtgd scnnymltyn

1861 kvenmtprdl gacidcsarh inaqvakshn ialiwnvkdf mslseqlrkq irsaakknnl

1921 pfkltcattr qvvnvvttki alkgg

Yellow: Luminal domain

Blue: Transmembrane domain

Green: Cytoplasmic domain 
C

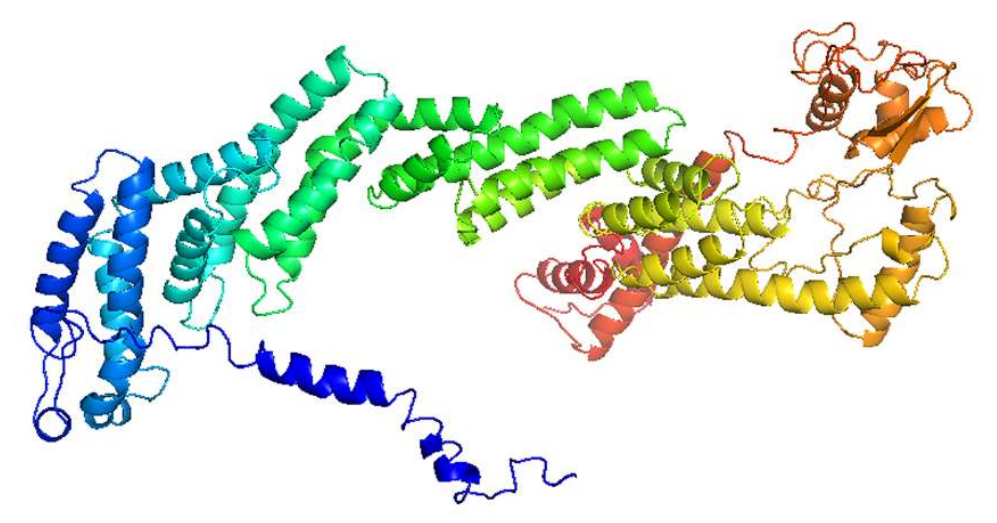

Fig.3. The topology of nsp4 of SARS-CoV-2. (A) Topology of nsp4 is determined using Protter. (B) The domains (cytoplasmic, transmembrane, luminal) of nsp4. (C) The predicted nsp4 structure of SARS-CoV-2 (ribbon diagram) determined using the software I-TASSER.

A

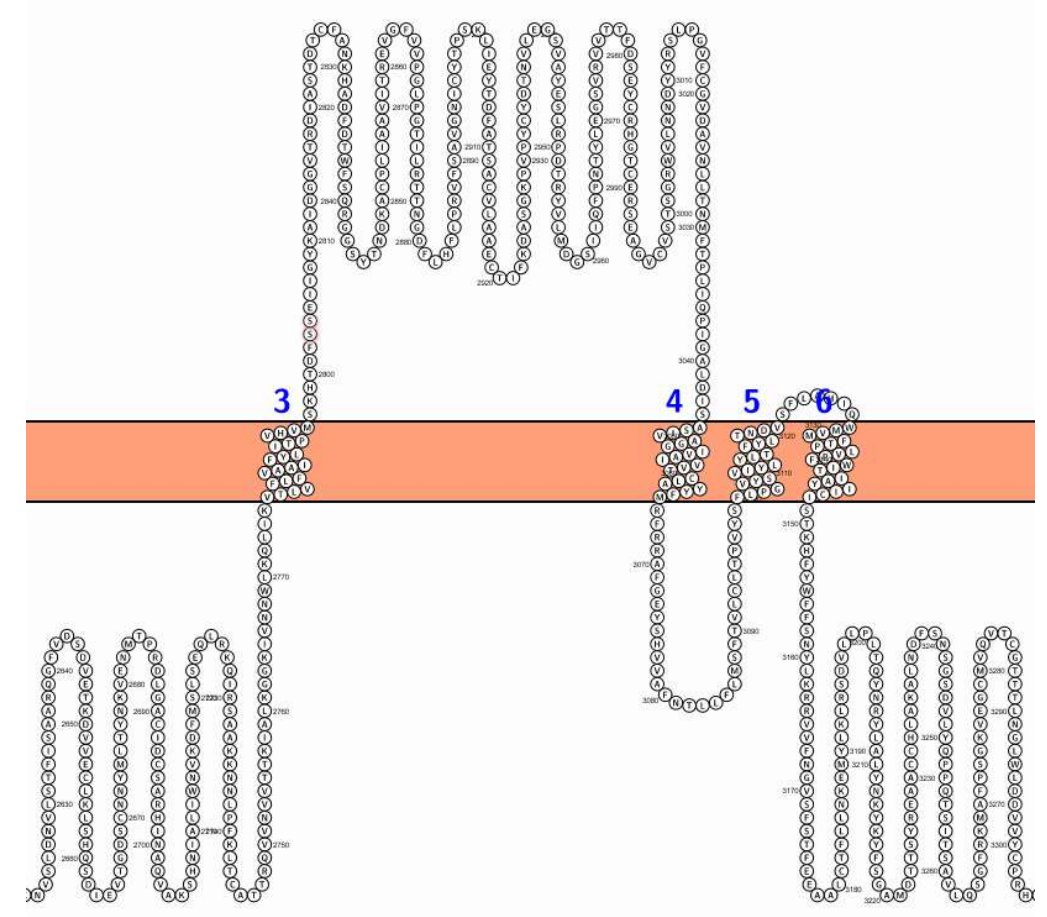


B

1 kivnnwlkql ik vtlvflfv aaifylitpv hvm skhtdfs seiigykaid ggvtrdiast 61 dtcfankhad fdtwfsqrgg sytndkacpl iaavitrevg fvvpglpgti lrttngdflh 121 flprvfsavg nicytpskli eytdfatsac vlaaectifk dasgkpvpyc ydtnvlegsv 181 ayeslrpdtr yvlmdgsiiq fpntylegsv rvvttfdsey crhgtcerse agvcvstsgr 241 wvlnndyyrs lpgvfcgvda vnlltnmftp liqpigaldi s asivaggiv aivvtclayy $301 \mathrm{fm}$ rfrrafge yshvvafntl lflmsftvlc ltpvys flpg vysviylylt fyltndv sfl 361 ahiq wmvmft plvpfwitia yiici stkhf ywffsnylkr rvvfngvsfs tfeeaalctf 421 llnkemylkl rsdvllpltq ynrylalynk ykyfsgamdt tsyreaacch lakalndfsn 481 sgsdvlyqpp qtsitsavlq

Yellow: Luminal domain

Blue: Transmembrane domain

Green: Cytoplasmic domain

C

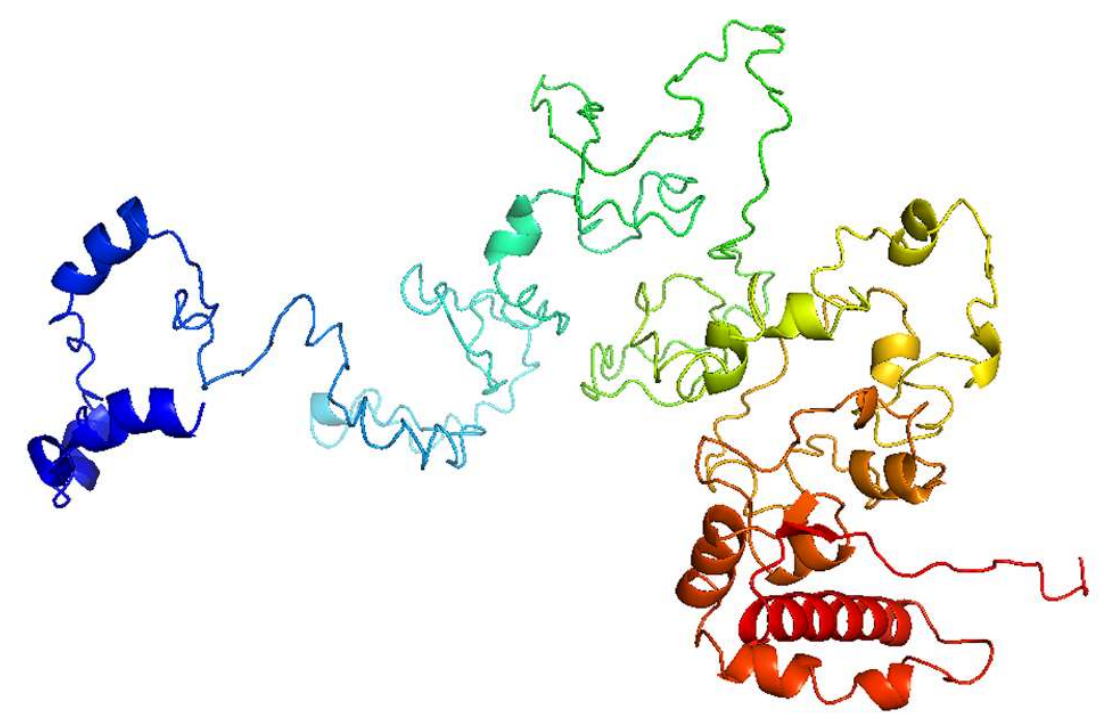


Fig.4. The topology of nsp6 of SARS-CoV-2. (A) Topology of nsp6 is determined using Protter. (B) The domains (cytoplasmic, transmembrane, luminal) of nsp6. The predicted nsp6 protein structure of SARS-CoV-2 (ribbon diagram) determined using the software ITASSER.

A

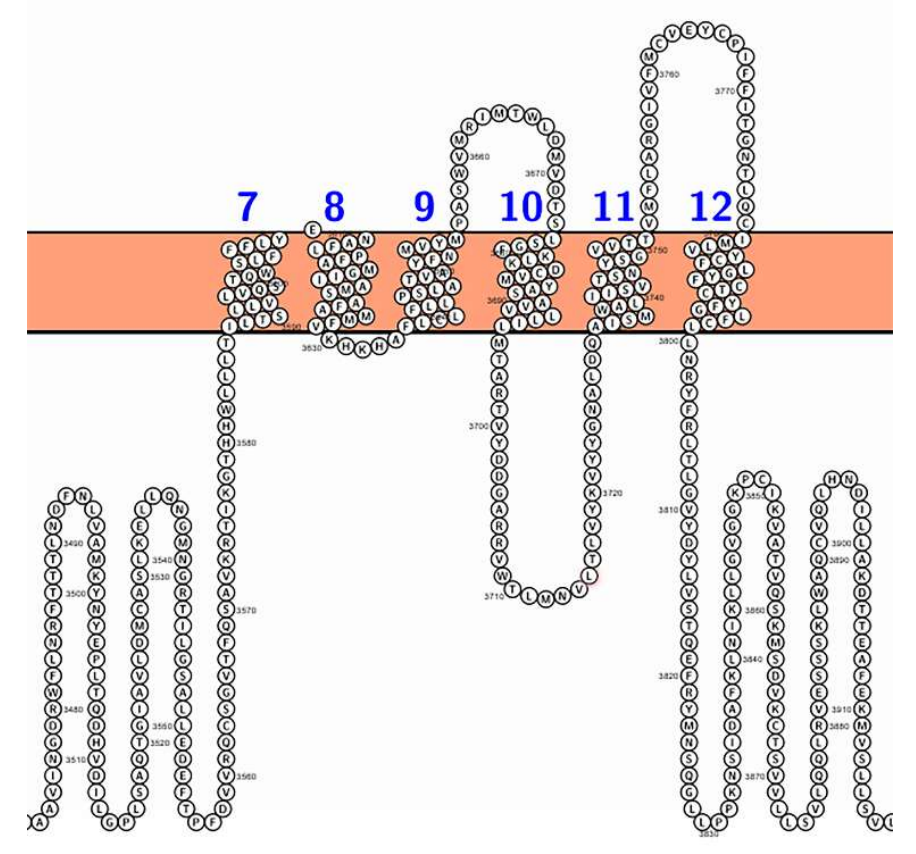

B

1 savkrtikgt hhwlllt ilt sllvlvqstq wslffflyen aflpfamgii amsafammfv

61 khkha flclf llpslatvay fnmvym pasw vmrimtwldm vdts lsgfkl kdcvmyasav

121 vllil mtart vyddgarrvw tlmnvltlvy kvyygnaldq aismwaliis vtsnysgvvt

$181 \mathrm{t}$ vmflargiv fmcveycpif fitgntlqc i mlvycflgyf ctcyfglfcl lnryfrltlg

241 vydylvstqe frymnsqgll ppknsidafk lnikllgvgg kpcikvatvq

Yellow: Luminal domain

Blue: Transmembrane domain

Green: Cytoplasmic domain 
C

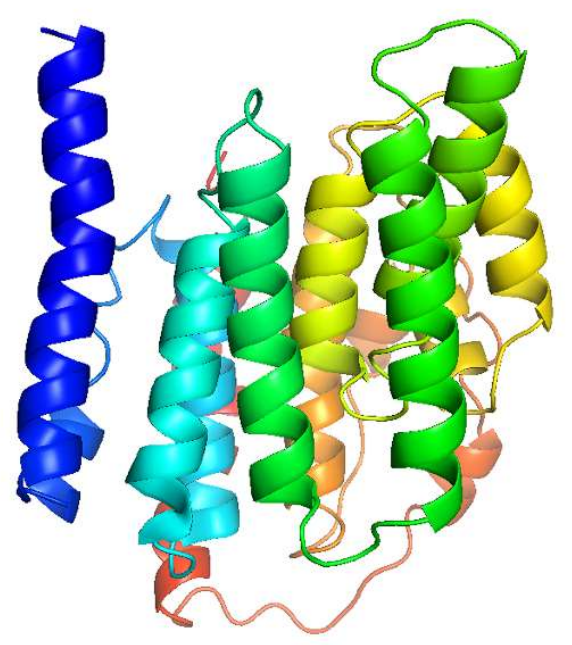

Fig.5. The topology of Orf3a of SARS-CoV-2. (A) The topology of Orf3a of SARS-CoV determined using Protter. (B) The SARS-CoV-2 and SARS-CoV Orf3a protein sequences are aligned using ClustalW. (C) The domains (cytoplasmic, transmembrane, luminal) of Orf3a of SARS-CoV-2. (D) The predicted Orf3a protein structure of SARS-CoV-2 (ribbon diagram) determined using the software I-TASSER.

A

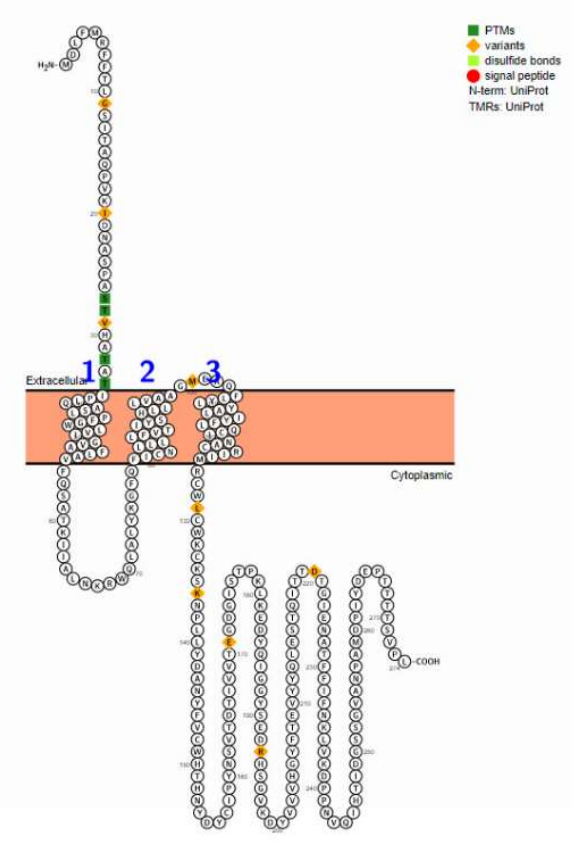


B

CLUSTAL o(1.2.4) multiple sequence alignment

BCI50534.1
SP|P59632.1|AP3A_SARS
BCI50534.1
SP|P59632.1|AP3A_SARS
BCI50534.1
SP|P59632.1|AP3A_SARS
BCI50534.1
SP|P59632.1|AP3A_SARS
BCI50534.1
SP|P59632.1|AP3A_SARS

MDLFMRIFTIGTVTLKQGEIKDATPSDFVRATATIPIQASLPFGWLIVGVALLAVFQSAS 60 MDLFMRFFTLGSITAQPVKIDNASPASTVHATATIPLQASLPFGWLVIGVAFLAVFQSAT 60

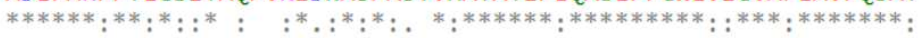

KIITLKKRWQLALSKGVHFVCNLLLLFVTVYSHLLLVAAGLEAPFLYLYALVYFLQSINF 120 KIIALNKRWQLALYKGFQFICNLLLLFVTIYSHLLLVAAGMEAQFLYLYALIYFLQCINA 120

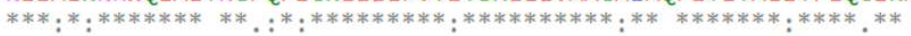

VRIIMRLWLCWKCRSKNPLLYDANYFLCWHTNCYDYCIPYNSVTSSIVITSGDGTTSPIS 180 CRIIMRCWLCWKCKSKNPLLYDANYFVCWHTHNYDYCIPYNSVTDTIVVTEGDGISTPKL 180

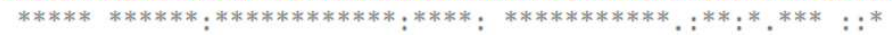

EHDYQIGGYTEKWESGVKDCVVLHSYFTSDYYQLYSTQLSTDTGVEHVTFFIYNKIVDEP 240 KEDYQIGGYSEDRHSGVKDYVVVHGYFTEVYYQLESTQITTDTGIENATFFIFNKLVKDP 240

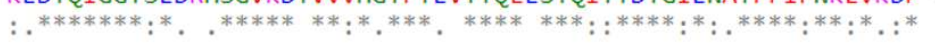

EEHVQIHTIDGSSGVVNPVMEPIYDEPTTTTSVPL 275 P-NVQIHTIDGSSGVANPAMDPIYDEPTTTTSVPL 274

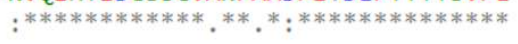

\section{BCI50534.1: Orf3a SARS-CoV-2 \\ P59632.1: Orf3a SARS-CoV \\ Percent identity: $\mathbf{7 3 . 0 0}$}

C

1 mdlfmrifti gtvtlkqgei kdatpsdfvr atat ipiqas lpfgwlivgv allav fqsas

61 kiitlkkrwq lalskgvh fv cnllllfvtv yshlllvaag leapflylya lvyflqsinf 121 vriim rlwlc wkcrsknpll ydanyflcwh tncydycipy nsvtssivit sgdgttspis 181ehdyqiggyt ekcesgvkdc vvlhsyftsd yyqlystqls tdtgvehvtf fiynkivdep 241eehvqihtid gssgvvnpvm epiydepttt tsvpl

Yellow: Luminal domain

Blue: Transmembrane domain

Green: Cytoplasmic domain 
D

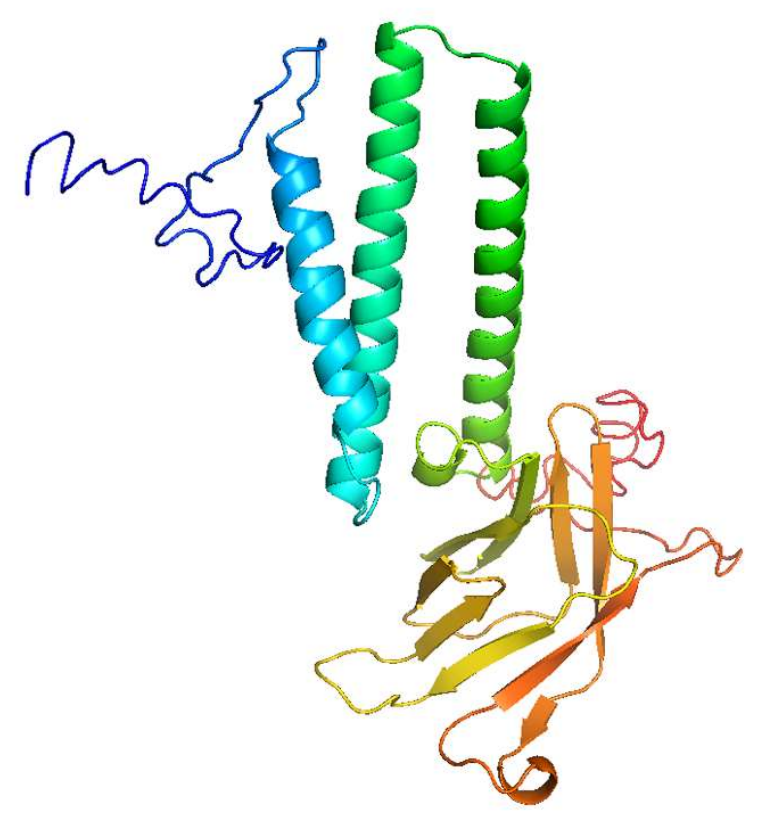

